\title{
Leucemia promielocítica aguda. Resultados del protocolo terapéutico LPA2000, Programa Nacional de Cáncer del Adulto (PANDA), Ministerio de Salud, Chile
}

\author{
MARÍA SOLEDAD UNDURRAGA ${ }^{1, \mathrm{a}}$, BÁRBARA PUGA ${ }^{1, \mathrm{a}}$, \\ MARÍA ELENA CABRERA ${ }^{1, \mathrm{~b}}$, TAMARA PALMA ${ }^{1, \mathrm{c}}$, \\ ALBERTO ROSSLE ${ }^{2, \mathrm{~d}}$, CARMEN GLORIA VERGARA ${ }^{3, \mathrm{~d}}$, \\ BERNARDITA ROJAS ${ }^{4, \mathrm{~d}}$, AUGUSTO ASPILLAGA ${ }^{5, \mathrm{~d}}$
}

\section{Acute promyelocytic leukemia. Results of the Chilean protocol LPA2000}

Background: The current recommendations for treatment of patients with newly diagnosed acute promyelocytic leukemia (APL) include all-trans-retinoic acid (ATRA) and anthracycline based chemotherapy. Aim: To evaluate the results of the Chilean protocol following the LPA99 regimen of the Spanish PETHEMA group, except for the replacement of Idarubicin by Daunorubicin. Patients and Methods: Induction consisted of Daunorubicin $45 \mathrm{mg} / \mathrm{m}^{2}$ on days 2, 4, 6 and 8 plus ATRA $45 \mathrm{mg} / \mathrm{m}^{2}$ daily until complete remission. Patients in complete remission (CR) received three monthly chemotherapy courses: Daunorubicin $45 \mathrm{mg} / \mathrm{m}^{2} / d / 4$ days i.v. and ATRA $45 \mathrm{mg} / \mathrm{m}^{2} / \mathrm{d} / 15$ days p.o. (course no. 1); Mitoxantrone $10 \mathrm{mg} / \mathrm{m}^{2} / \mathrm{d} / 5$ days i.v. and ATRA $45 \mathrm{mg} / \mathrm{m}^{2} / \mathrm{d} / 15$ days p.o. (course no. 2); Daunorubicin $60 \mathrm{mg} / \mathrm{m}^{2} / \mathrm{d} /$ day 1 i.v. in the low risk group, and 1 and 2 in the intermediate-high risk groups and ATRA $45 \mathrm{mg} / \mathrm{m}^{2} / \mathrm{d} / 15$ days p.o. (course no. 3). Maintenance therapy consisted of mercaptopurine $90 \mathrm{mg} / \mathrm{m}^{2} / d$ p.o., methotrexate $15 \mathrm{mg} / \mathrm{m}^{2} / \mathrm{wk}$ p.o. and, ATRA intermittently, $45 \mathrm{mg} / \mathrm{m}^{2} / d$ p.o. for 15 days every three months. Results: Between January 2000 and December 2005, 56 patients with newly diagnosed APL from 10 centers were enrolled. A total of 46 patients achieved CR (85\%), 8 (15\%) died of early complications, seven patients relapsed, with a $16 \%$ relapse risk at three years. The 5-year Kaplan-Meier estimates of overall survival and relapse-free survival were $64 \%$ and $84 \%$ respectively. Conclusions: These data indicate that this protocol has a good antileukemic effect but further reduction of early death and relapse, especially in the high risk group is needed.

(Rev Med Chile 2013; 141: 1231-1239).

Key words: Daunorubicin; Leukemia, myeloid, acute; Mitoxantrone; Tretinoin.

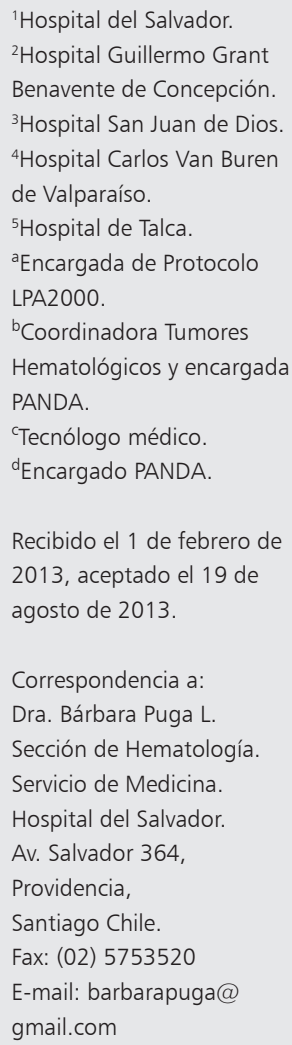

U no de los mayores avances en leucemia aguda en los últimos 15 años se ha producido en la leucemia promielocítica aguda (LPA) que constituye 10 a 15\% de las leucemias mieloides agudas (LMA) con una mayor incidencia $(24 \%)$ en población latina ${ }^{1}$. Se presenta con mayor frecuencia en gente joven. La LPA, efecto de una traslocación específica $t(15 ; 17)$ que interfiere con la acción de diferenciación de los retinoides a nivel de los promielocitos, se caracteriza por una coagulopatía de consumo asociada a una elevada mortalidad precoz y alta sensibilidad a las antraciclinas $^{2-4}$. En la actualidad, con la incorporación concomitante del ácido all-trans retinoico (ATRA) 
al tratamiento de quimioterapia, la implementación de medidas de soporte durante la inducción y el tratamiento de mantención, es la leucemia aguda de mejor pronóstico en el adulto, con una curación superior a $80 \%{ }^{5-9}$.

En Chile, en el año 1988 se dio inicio al Programa Nacional de Cáncer del Adulto (PANDA), responsable de la definición de los tratamientos de los diferentes tipos de cáncer para $80 \%$ de la población chilena, beneficiaria de la salud pública. Desde esa fecha, se reconocen tres períodos en el tratamiento de la LPA en nuestro país: 1) Desde 1988 hasta el año 1993, en que la LPA fue tratada con citarabina y daunorrubicina en un ciclo de inducción y tres consolidaciones, al igual que otras leucemias mieloblásticas agudas, con una sobrevida global (SG) a 5 años de 6\%;2) La incorporación del ATRA en 1993 en forma no estandarizada (uso exclusivo o consecutivo a quimioterapia convencional) significó un aumento de la sobrevida global a 5 años, que alcanzó 25\% (Figura 1); 3 ) El año 2000, basados en la exitosa experiencia del grupo español PETHEMA con el protocolo LPA99 que alcanzó una SG de $85 \%$, se inició un protocolo para este subtipo de leucemia, LPA2000 ${ }^{10,11}$. En este protocolo, además de excluir el uso de citarabina, se reemplazó la idarrubicina por daunorrubicina por no existir evidencia que mostrara diferente efectividad entre ellas ${ }^{12-16}$. Se estandarizó el tratamiento de soporte en la inducción según protocolo nacional, especificándose las medidas preventivas y terapéuticas para coagulopatía, síndrome de ATRA (SAR) y neutropenia febril de alto riesgo ${ }^{17,18}$.

El objetivo del presente trabajo fue analizar las características clínicas de la LPA en población

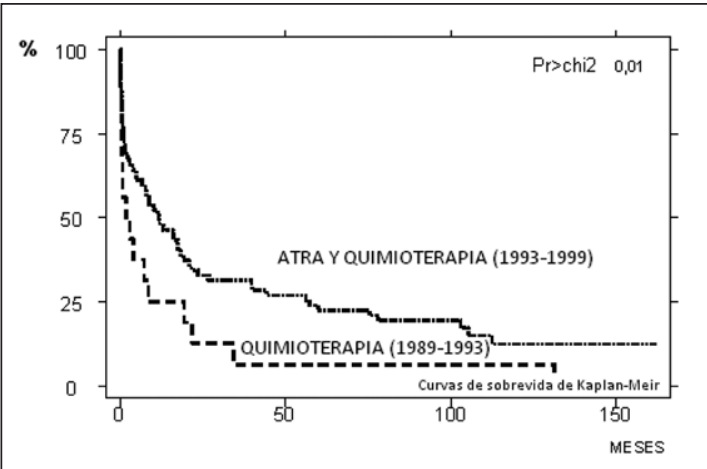

Figura 1. Sobrevida global en LPA Chile, comparación histórica 1988-1999. chilena y evaluar los resultados del protocolo LPA2000, comparándolos con los del LPA99 del grupo español PETHEMA.

\section{Pacientes y Método}

Entre el $1^{\circ}$ de enero de 2000 y diciembre de 2005 se registraron 643 casos de leucemia aguda en mayores de 15 años en los centros de cáncer del adulto del sistema público de salud de Chile. De estos, 417 casos fueron leucemia mieloide aguda (LMA) de los cuales $56(13,4 \%)$ correspondieron a LPA. La distribución de los casos por centro hospitalario se muestra en la Figura 2. La morfología de todos los casos fue revisada en forma centralizada. Los estudios de inmunofenotipo y citogenética se realizaron en los laboratorios de referencia nacional del Hospital del Salvador. El estudio de biología molecular fue realizado en un laboratorio externo de referencia.

\section{Diagnóstico}

Para el diagnóstico de LPA, se consideró la presencia de la traslocación $\mathrm{t}(15 ; 17)$ por citogenética o del gen $P M L / R A R a$ por biología molecular. En ausencia de estos estudios, se consideró también aquellos con morfología LMA-M3 asociada o no a inmunofenotipo sugerente. Los 56 pacientes ingresados al protocolo fueron clasificados según grupos de riesgo de acuerdo a la descripción de Sanz ${ }^{11}$.

\section{Tratamiento}

Todos los pacientes con diagnóstico de LPA recibieron tratamiento según protocolo LPA2000 (Tabla 1) previo consentimiento. El tratamiento de soporte en la inducción consistió en transfusiones de plaquetas para mantener recuentos superiores a $30.000 \mathrm{x} \mathrm{mm}^{3}$, crioprecipitados para mantener fibrinógeno sobre $100 \mathrm{mg} / \mathrm{dl}$, plasma fresco congelado para mantener tiempo de protrombina (PT) y tromboplastina parcial activado (TTPK) en rangos normales. Se administró ácido tranexámico $100 \mathrm{mg} / \mathrm{Kg} /$ día i.v. en infusión continua hasta recuperación de la coagulopatía y plaquetas sobre $20.000 \times \mathrm{mm}^{3}$. Como profilaxis del SAR se utilizó prednisona $0,5 \mathrm{mg} / \mathrm{kg} /$ día p.o., días 1 al 14 . En caso de sospecha de SAR se usó dexametasona $10 \mathrm{mg}$ c/12 h i.v. por tres días. La suspensión de ATRA se indicó en casos de SAR severo, para ser reiniciado después de superada la complicación. 


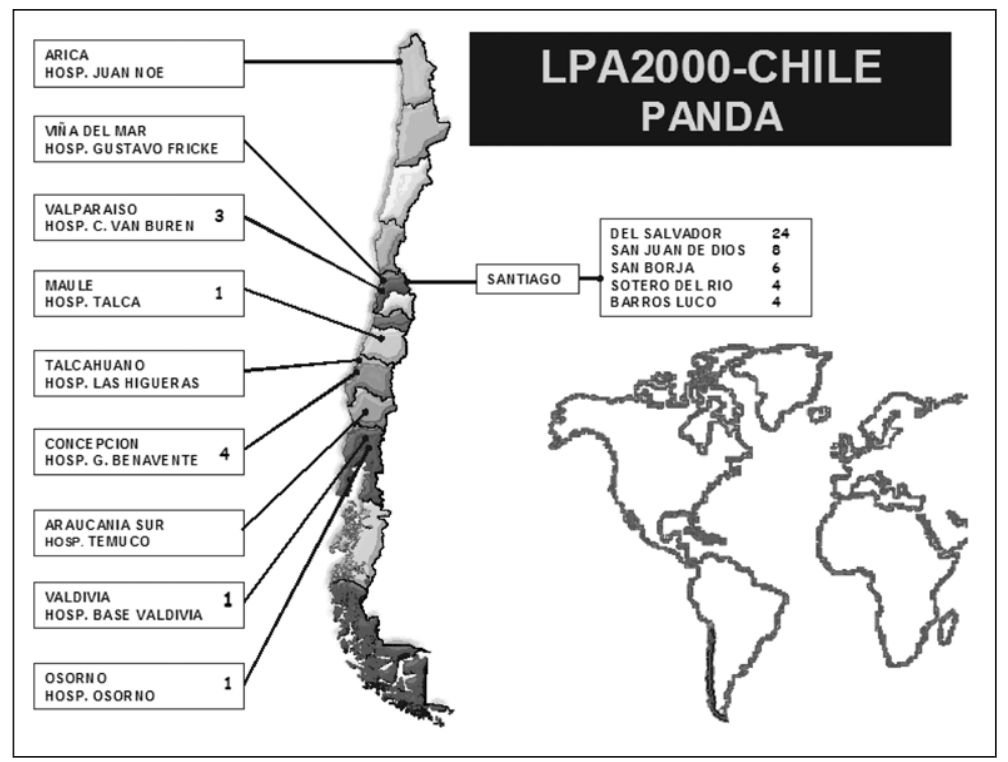

Figura 2. Distribución geográfica de los centros PANDA acreditados para el tratamiento de leucemias agudas y número de casos de LPA aportados por cada uno.

Tabla 1. Protocolo terapéutico LPA2000

\begin{tabular}{|c|c|c|c|}
\hline \multirow[t]{2}{*}{ Inducción } & \multicolumn{3}{|c|}{ Daunorrubicina (DNR) $45 \mathrm{mg} / \mathrm{m}^{2} /$ día, días 2-4-6-8 + ATRA $45 \mathrm{mg} / \mathrm{m}^{2} / \mathrm{hasta}$ RC } \\
\hline & Riesgo bajo & Riesgo intermedio & Riesgo alto \\
\hline Consolidación 1 & $\begin{array}{l}\text { DNR } 30 \mathrm{mg} / \mathrm{m}^{2} / \text { día, días } 1-4 \\
+ \text { ATRA } 45 \mathrm{mg} / \mathrm{m}^{2} / 15 \text { días }\end{array}$ & $\begin{array}{l}\text { DNR } 30 \text { mg/m²/día, días } 1-4 \\
+ \text { ATRA } 45 \mathrm{mg} / \mathrm{m}^{2} / 15 \text { días }\end{array}$ & $\begin{array}{l}\text { DNR } 30 \mathrm{mg} / \mathrm{m}^{2} / \text { día, días } 1-4+\text { ATRA } \\
45 \mathrm{mg} / \mathrm{m}^{2} / 15 \text { días + profilaxis sistema } \\
\text { nervioso central (SNC) (Metotrexato } \\
\text { (MTX), ARA-C, Betametsona) }\end{array}$ \\
\hline Consolidación 2 & $\begin{array}{l}\text { Mitoxantrona (MTZ) } 10 \mathrm{mg} / \\
\mathrm{m}^{2} / \mathrm{día} \text {, días } 1-4+\text { ATRA } 45 \\
\mathrm{mg} / \mathrm{m}^{2} / 15 \text { días }\end{array}$ & $\begin{array}{l}\text { MTZ } 10 \mathrm{mg} / \mathrm{m}^{2} / \text { día, días } 1-5 \\
+ \text { ATRA } 45 \mathrm{mg} / \mathrm{m}^{2} / 15 \text { días }\end{array}$ & $\begin{array}{l}\text { MTZ } 10 \mathrm{mg} / \mathrm{m}^{2} / \text { día, días } 1-5+\text { ATRA } \\
45 \mathrm{mg} / \mathrm{m}^{2} / 15 \text { días + profilaxis SNC } \\
\text { (MTX,ARA-C, Betametsona) }\end{array}$ \\
\hline Consolidación 3 & $\begin{array}{l}\text { DNR } 60 \mathrm{mg} / \mathrm{m}^{2} / \text { día } 1+ \\
\text { ATRA } 45 \mathrm{mg} / \mathrm{m}^{2} / 15 \text { días }\end{array}$ & $\begin{array}{l}\text { DNR } 60 \text { mg/m²/día, días } 1 \text { y2 } \\
+ \text { ATRA } 45 \mathrm{mg} / \mathrm{m}^{2} / 15 \text { días }\end{array}$ & $\begin{array}{l}\text { DNR } 60 \mathrm{mg} / \mathrm{m}^{2} / \text { días } 1 \text { y } 2+\text { ATRA } \\
45 \mathrm{mg} / \mathrm{m}^{2} / 15 \text { días + profilaxis SNC } \\
\text { (MTX,ARA-C, Betametasona) }\end{array}$ \\
\hline Mantención & \multicolumn{3}{|c|}{$\begin{array}{l}\text { Mercaptopurina } 90 \mathrm{mg} / \mathrm{m}^{2} / \mathrm{día}+\text { MTX } 10 \mathrm{mg} / \mathrm{m}^{2} / \mathrm{semana}+\text { ATRA } 45 \mathrm{mg} / \mathrm{m}^{2} / 15 \text { días intermitente } \\
\text { cada } 3 \text { meses por } 2 \text { años }\end{array}$} \\
\hline
\end{tabular}

\section{Seguimiento}

Se realizó seguimiento clínico y de laboratorio mensual el primer año y trimestral hasta la finalización de la fase de mantención. Después de finalizado el tratamiento, el control fue semestral. El seguimiento de la enfermedad mínima residual (EMR) por biología molecular se inició después de finalizada la tercera consolidación y cada tres meses los primeros 2 años.

\section{Definición de Criterios de Evaluación}

Se definió remisión completa $(\mathrm{RC})$ a la presencia de un hemograma normal y un aspirado medular con menos de 5\% de blastos en el día 30 de la inducción; mortalidad precoz (MP), al fallecimiento ocurrido en el primer mes de tratamiento y después de $48 \mathrm{~h}$ de iniciada la inducción; recaída $(\operatorname{Rec})$ a la presencia de más de 5\% de blastos en médula ósea o la detección del gen PML/RARa, en dos determinaciones sucesivas, separadas por al menos 2 semanas, después de haberse negativizado. Sobrevida global (SG) se midió desde el inicio del tratamiento hasta la muerte por cualquier causa o último control. Sobrevida libre de recaída (SLR) se midió desde la RC hasta la fecha de Rec. 


\section{Análisis estadísticos}

Las curvas de SLR y SG se estimaron por el método de Kaplan-Meier y la comparación de ellas fue hecha a través del test de Log-Rank. La comparación de variables dicotómicas se realizó mediante la prueba de independencia de $\chi^{2}$ o prueba de Fisher. La significación fue de 5\%.

\section{Resultados}

Se consideraron 56 casos de LPA, rango de edad entre 15 y 78 años (mediana 34), 70\% menores de 40 años, predominio de mujeres (relación 1,7/1). La clasificación de riesgo fue: bajo (RB) 5 casos (9\%), intermedio (RI) 39 casos (70\%) y alto (RA) 12 casos (21\%).

El diagnóstico de LPA se realizó por citogenética y/o RT-PCR en 38 casos (68\%), por morfología e inmunofenotipo (CD34 (-); DR (-), CD13 (+), CD33 (+) en 11 casos (20\%) y por morfología con cuadro clínico característico en 7 casos (12\%). En todos los pacientes con estudio citogenético se observó traslocación t $(15 ; 17)$, siendo en $28 / 35$ (80\%) el único hallazgo. El 20\% restante se asoció a alteraciones adicionales, siendo la trisomía 8 la más frecuente.

El inmunofenotipo, por citometría de flujo, fue característico en $42 / 46$ casos estudiados (92\%) con negatividad de CD34 y HLA-DR. El antígeno CD2 fue positivo en 4 pacientes, 3 de los cuales presentaron leucocitosis, morfología microgranular y expresión de CD34 (Tabla 2).

Dos pacientes fallecieron antes de iniciar tratamiento. De los 54 pacientes tratados, $46(85 \%)$ lograron RC. Ocho pacientes (15\%) fallecieron
Tabla 2. Características de laboratorio al diagnóstico y definición de riesgo en 56 casos de LPA

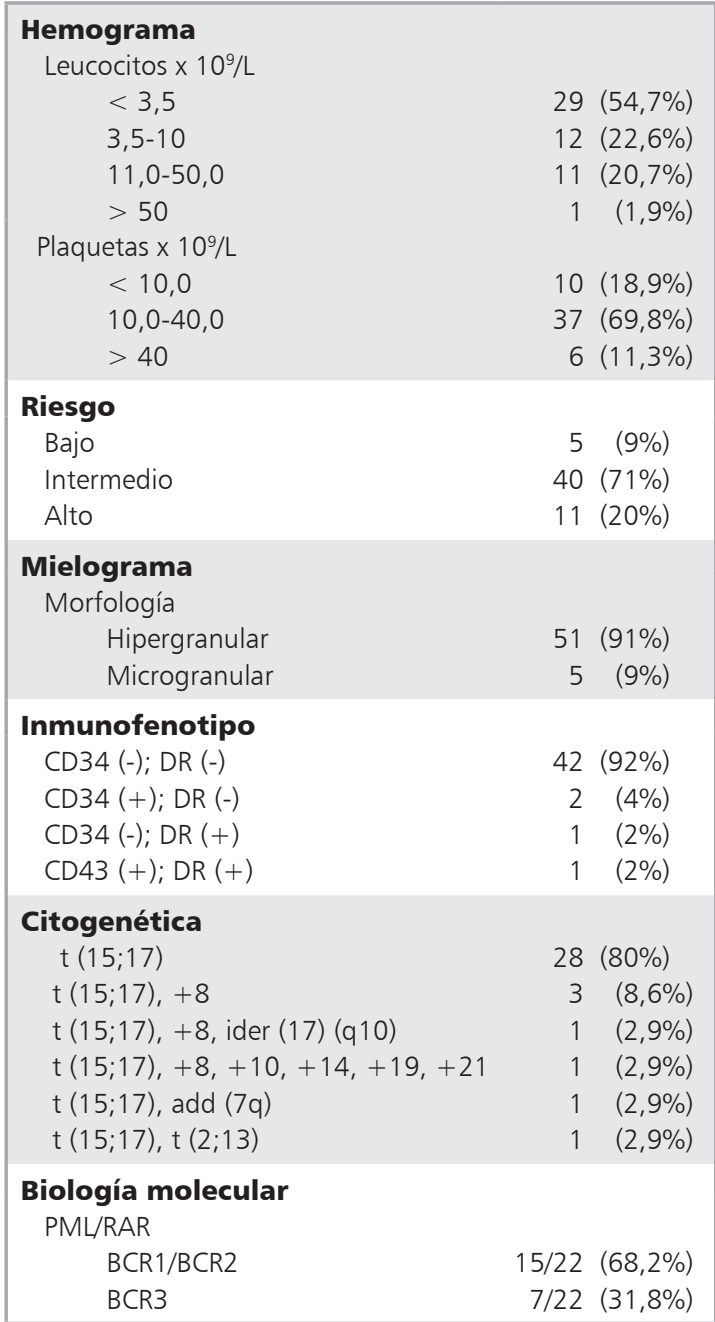

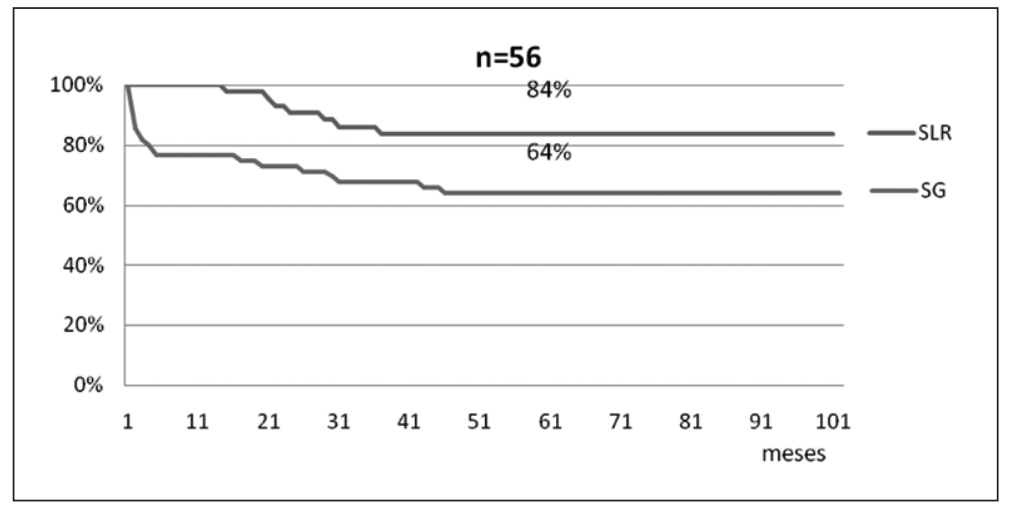

Figura 3. Sobrevida global y libre de recaída. Protocolo LPA 2000, Ministerio de Salud, PANDA. Chile 2000-2010. 

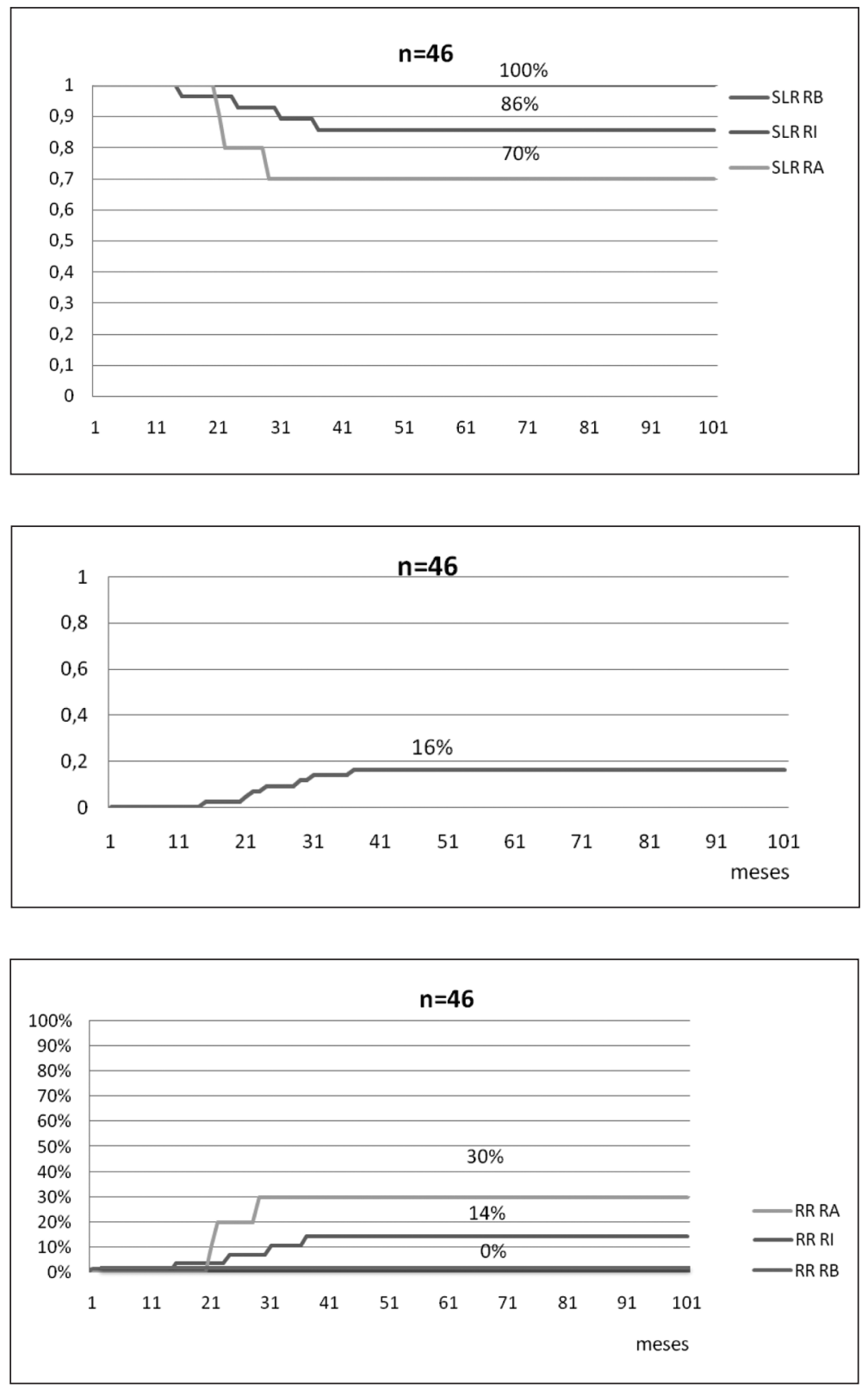

Figura 4. Sobrevida libre de recaída según riesgo. Protocolo LPA 2000, Ministerio de Salud, PANDA. Chile 2000-2010.

Figura 5. Riesgo de recaída. Protocolo LPA 2000, Ministerio de Salud, PANDA. Chile 2000-2010.

Figura 6. Riesgo de recaída según riesgo. Protocolo LPA 2000, Ministerio de Salud, PANDA. Chile 2000-2010. durante la quimioterapia de inducción. Cuatro de ellos, en relación a cuadro séptico, 2 a cuadro hemorrágico y 2 en asociación a síndrome de ATRA. De los 46 pacientes que lograron RC, 4 abandonaron tratamiento sin completar las consolidaciones, 1 de ellos, por intolerancia al tratamiento. De los 46 pacientes que alcanzaron RC, 3 fallecieron por cuadro séptico en período de aplasia durante quimioterapia de consolidación.
Se observó recaída en 7 pacientes, cuatro de RI y 3 de RA. La mediana de seguimiento fue 49 meses. La SG a 5 años para los 54 pacientes tratados fue 64\% (Figura 3). La SLR a 5 años en el grupo de pacientes que alcanzó RC fue $84 \%$ (RB 100\%, RI $86 \%$ y RA $70 \%)(\mathrm{p}<0,05)$ (Figura 4$)$. El riesgo de recaída acumulado a 3 años fue $0 \%$ en RB, $14 \%$ en RI y $30 \%$ en RA (Figura 5 y 6 ). No se detectaron recaídas después de los 36 meses. 


\section{Discusión}

Las características epidemiológicas del grupo de pacientes chilenos son similares a las descritas en la literatura con predominio de pacientes jóvenes y riesgo intermedio. La literatura señala que la proporción de LPA dentro de la LMA suele ser mayor en países latinos, lo que no se demuestra en nuestra población (13\%). Una razón podría ser que existiera un subregistro a nivel nacional en una patología con alta mortalidad al momento del diagnóstico o al número reducido de casos.

La sospecha diagnóstica se plantea con un cuadro hemorragíparo mucocutáneo con o sin trombocitopenia severa, morfología LMA-M3 e inmunofenotipo mieloide con CD34 y DR negativos. Estos elementos permiten plantear el diagnóstico de LPA e iniciar precozmente tratamiento con ATRA y medidas de soporte para el control de la coagulopatía, antes de la confirmación por biología molecular y/o el estudio citogenético. Así, el inmunofenotipo es un examen de gran utilidad ya que es característico en este subtipo de leucemia aguda y su informe puede conocerse antes de 24 $\mathrm{h}^{19,20}$. En nuestro estudio, 92\% presentó negatividad para ambos marcadores en forma similar a lo observado en la literatura. Asimismo, la expresión de CD34 y/o DR en asociación a expresión de CD2 es altamente sugerente de variedad microgranular, leucocitosis y mal pronóstico ${ }^{21}$. En nuestro estudio, 2 pacientes con estas características fallecieron antes de iniciar tratamiento y otro mostró persistencia de EMR después de varias líneas terapéuticas.

La asociación de $\mathrm{t}(15 ; 17)$ con otras alteraciones citogenéticas encontradas en $20 \%$ de los pacientes, coincide con los datos de otros grupos. A diferencia de otras leucemias agudas, en la LPA no se ha demostrado asociación con mal pronóstico. En nuestro estudio no fue posible determinar la importancia pronóstica de estas alteraciones citogenéticas por tratarse de un grupo pequeño de pacientes ${ }^{22}$.

La mortalidad precoz de 15\% aún es elevada, aunque inferior a lo reportado por otros países en vías de desarrollo de $32 \%{ }^{23-25}$. Esto podría estar relacionado a la geografía chilena, con zonas de difícil acceso a los servicios de salud y a la diferencia en las capacidades instaladas entre los distintos centros.

El pronóstico de la LPA en Chile ha mejorado significativamente en los últimos 16 años con SG

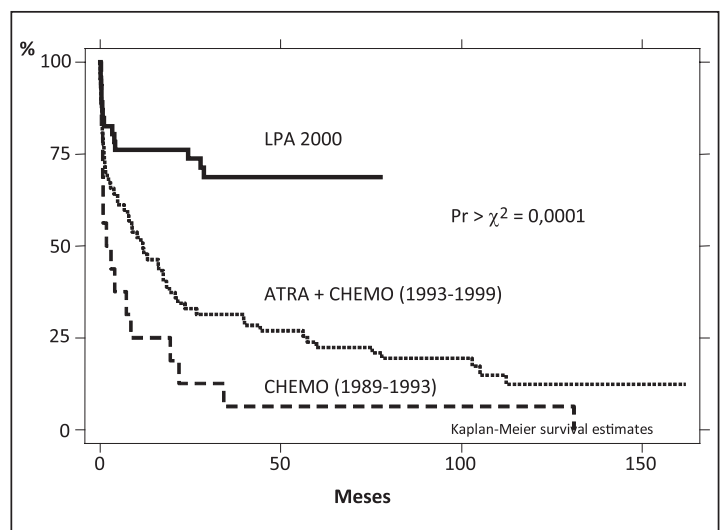

Figura 7. Sobrevida global en LPA Chile, comparación histórica 1988-2005.

de $6 \%$ el año 1993 a $67 \%$ con el presente protocolo (Figura 7). La estandarización del tratamiento y de las medidas de soporte indicadas en el protocolo LPA2000 ha sido fundamental en estos logros.

Aunque la SLR de $84 \%$ es similar, a lo referido por el grupo PETHEMA y otros grupos europeos, la SG de 64\% es francamente inferior al $85-97 \%$ que reportan a 3 años ${ }^{9-11,26-28}$. Esta diferencia se explica por una elevada mortalidad relacionada a tratamiento tanto en inducción como en consolidación.

\section{Conclusión}

LPA2000 es un tratamiento efectivo que ha mejorado significativamente la sobrevida de la LPA en Chile (Figura 7). La reciente centralización del tratamiento de las leucemias agudas en hospitales acreditados y su incorporación al programa gubernamental de Garantías Explícitas en Salud (GES) en julio de 2007, permitirán mejorar nuestros resultados al reducir la mortalidad relacionada a quimioterapia y el riesgo de recaída en una patología con alta tasa de curación. El ingreso de las leucemias agudas al GES garantiza la oportunidad de atención, el estudio morfológico, inmunofenotípico, citogenético y molecular de esta patología, la calidad del tratamiento así como el seguimiento molecular. La incorporación al protocolo del Consorcio Internacional en Leucemia Promielocítica Aguda para países en vías de desarrollo, IC-APL, que considera la intensificación de la consolidación en el grupo de RA, debiera reflejarse a futuro 
Leucemia promielocítica aguda. Resultados del PANDA - M. S. Undurraga et al

en una mejoría de la $\mathrm{SG}^{29-31}$. Confiamos que la implementación de estas medidas nos permita dar inicio a un cuarto período en la historia de la LPA en Chile garantizando las mejores posibilidades de curación.

Agradecimientos: Los siguientes centros y personas participaron con pacientes en este estudio: Arica: Dra. Cecilia Hales D; Gustavo Fricke: Drs. Carlos Merino M, Ismelda Toro; Carlos Van Buren: Drs. Pedro Meneses C, Bernardita Rojas M; Talca: Dr. Augusto Aspillaga M; Concepción: Drs. Annemarie Fahrenkrog C, Alberto Rossle S, Guido Bancalari B; Talcahuano: Dra. Jacqueline Oliva L; Temuco: Drs. Cecilia Vittini de R, Eduardo Yáñez R, Ana Peña Q, Consuelo Rodríguez B; Valdivia: Drs. Álvaro León R, Lilian Pilleux C, Susana Calderón A, Pilar Salas T, Blas Lesina B; Osorno: Drs. Janet Rosas H, M. Luisa González; Hospital San Juan de Dios: Drs. Carmen Gloria Vergara, Karina Peña N, Lucía Puente S; Hospital del Salvador: Drs. M. Elena Cabrera C, M. Soledad Undurraga S, Ana María Gray H, Bárbara Puga L, Carolina Guerra C; Hospital San Borja Arriarán: Drs. Hernán García L, Ana María Riquelme F; Hospital Barros Luco Trudeau: Drs. Vivian Lois V, Isaac Con R, Lina Muñoz R, M. Isabel Bustos G, Juan Luis Castro G, Federico Liendo P, Guido Osorio S; Hospital Sótero del Río: Drs. Hernán Rojas R, Denis Suárez.

\section{Referencias}

1. Douer D, Preston-Martin S, Chang E, Nichols PW, Watkins KJ, Levine AM. High frequency of acute promyelocytic leukemia among Latinos with acute myeloid leukemia. Blood 1996; 87: 308-13. Disponible en www. pubmed.com (consultado el 8 de noviembre de 2012).

2. Miller WH Jr, Kakisuka A, Frankel SR, Warrell RP, Deblasio A, Levine K, et al. Reverse transcription polymerase reaction for the rearranged retinoic acid receptor $\alpha$ clarifies diagnosis and detects minimal residual disease in acute promyelocytic leukemia. Proc Natl Acad Sci USA 1992; 89: 2694-8. Disponible en www.pubmed.com (consultado el 8 de noviembre de 2012).

3. Tallman M, Brenner B, De La Serna J, Dombret H, Falanga A, Kwaan HC, et al. Meeting Report. Acute Promyelocytic Leukemia-associated coagulopathy, january 21, 2004, London, United Kingdom. Leuk Res 2005; 29: 347-51. Disponible en www.pubmed.com (consultado el 8 de noviembre de 2012).
4. Bernard J, Weil M, Boiron M, Jacquillat C, Flandrin G, Gemon MF. Acute promyelocytic leucemia: results of treatment by daunorrubicin. Blood 1973; 41: 89-96. Disponible en www.pubmed.com (consultado el 8 de noviembre de 2012).

5. Fenaux P, Le deley MC, Castaigne S, Archimbaud E, Chomienne C, Link $\mathrm{H}$, et al. and the European APL 91 Group: Effect of all-trans retinoic acid in newly diagnosed acute promyelocytic leukemia. Results of a multicenter randomized trial. Blood 1993; 82: 3241-329. Disponible en www.pubmed.com (consultado el 8 de noviembre de 2012.

6. Frankel Sr, Eardley A, Heller G, Berman E, Miller Vm Jr, Dmitrvsky E, et al. All-trans retinoic acid for acute promyelocytic leukemia. Results of the New York study. Ann Intern Med 1994; 120: 278-86. Disponible en www. pubmed.com (consultado el 8 de noviembre de 2012).

7. Mandelli F, Diverio D, Avvisati G, Luciano A, Barbui T, Bernasconi C, et al. Molecular remission in PML/RARapositive acute promyelocytic leukemia by combined all-trans retinoic acid and idarubicin (AIDA) therapy. Blood 1997; 90: 1014-21. Disponible en www.pubmed. com (consultado el 8 de noviembre de 2012).

8. Tallman MS, Andersen JW, Schiffer CA, Appelbaum FR, Feusner JH, OGDEN A, et al. All-trans retinoic acid in acute promyelocytic leukemia. N Engl J Med 1997; 337: 1021-8. Disponible en www.pubmed.com (consultado el 8 de noviembre de 2012).

9. Fenaux P, Chastang C, Chevret S, Sanz M, Dombret $\mathrm{H}$, Archimbaud E, et al. A randomized comparison of ATRA followed by chemotherapy and ATRA plus chemotherapy, and the role of maintenance therapy in newly diagnosed acute promyelocytic leukemia. Blood 1999; 94: 1192-200. Disponible en www.pubmed.com (consultado el 8 de noviembre de 2012).

10. Sanz MA, Martín G, Rayón C, Esteve J, González M, Díaz-Mediavilla J, et al. A modified AIDA protocol with anthracycline-based consolidation results in high antileukemic efficacy and reduced toxicity in newly diagnosed PML/RARa positive acute promyelocytic leukemia. Blood 1999; 94: 3015-21. Disponible en www.pubmed. com (consultado el 8 de noviembre de 2012).

11. Sanz MA, Montesinos P, Vellenga E, Rayón C, De La Serna J, Parody R, et al. Risk-adapted treatment of acute promyelocytic leukemia with all-trans retinoic acid by PETHEMA group. Blood 2004; 103: 1237-43. Disponible en www.pubmed.com (consultado el 8 de noviembre de 2012).

12. Marty M, Ganem G, Fischer J, Flandrin G, Berger R, Schaison G, et al. Leucémie aigue promyelocitaire: étude retrospectiva de 119 malades traités par daunorubicine. 
Nouv Rev Fr Hematol 26: 371, 1984. Disponible en www.pubmed.com (consultado el 8 de noviembre de 2012).

13. Avvisati G, Mandelli F, Petti MC, Vegna ML, Spadea A, Liso $\mathrm{V}$, et al. Idarubicin (4demethoxydaunorubicin) as single agent for remission induction of previously untreated acute promyelocytic leukemia: A pilot study of the Italian cooperative group GIMEMA. Eur J Haematol 1990; 44: 257-60. Disponible en www.pubmed.com (consultado el 8 de noviembre de 2012).

14. Estey E, Thall PF, Pierce S, Kantarjian H, Keating M. Treatment of newly diagnosed acute promyelocytic leukemia without cytarabine. J Clin Oncol 1997; 15: 483-90. Disponible en www.pubmed.com (consultado el 8 de noviembre de 2012).

15. Hillman AL. Idarubicin more cost-effective than daunorubicin? Pharmacoeconomics 1992; 1: 69-70. Disponible en www.pubmed.com (consultado el 8 de noviembre de 2012).

16. Head D, Kopecky KJ, Weick J, Files JC, RYAN D, Foucar $\mathrm{K}$, et al. Effect of agressive daunomycin therapy on survival in acute promyelocytic leukemia. Blood 1995; 86: 1717-28. Disponible en www.pubmed.com (consultado el 8 de noviembre de 2012).

17. Undurraga MS. Protocolo de tratamiento leucemia promielocítica aguda. Protocolos Nacionales. Programa de Drogas Antineoplásica del adulto 2000-2001. Gobierno de Chile, Ministerio de Salud, Unidad de Cáncer.

18. Puga B, Puga I, Cabrera ME, Undurraga MS, Guerra C, Urrejola G, et al. High risk febrile netropenia. Experience in a public hospital: National Cancer Program (PANDA) at Hospital del Salvador (1991-2001). Rev Med Chile. 2003; 131: 1023-30. Disponible en www. pubmed.com (consultado el 8 de noviembre de 2012).

19. Kaleem Z, Crawford E, Pathan H, Jasper L, Covinsky MA, Johnson LR, et al. Flow cytometric analysis of acute leukemia. Diagnostic utility and critical analysis of data. Arch Pathol Lab Med 2003; 127: 42-8. Disponible en www. pubmed.com (consultado el 8 de noviembre de 2012).

20. Orfao A, Chillón MC, Bortoluci AM, López-Berges MC, García-Sanz R, González M, et al. The flow cytometric pattern of CD34, CD15 and CD13 expression in acute myeloblastic leukaemia is highly characteristic of the presence of PML-RAR gene rearrangements. Haematologica 1999; 84: 405-12. Disponible en www.pubmed. com (consultado el 8 de noviembre de 2012).

21. Kaito K, Katayama T, Masuoka H, Nishiwaki K, Sano K, Sekiguchi N, et al. CD2+ acute promyelocytic leucemia is associated with leukocytosis, variant morphology and poorer prognosis. Clin Lab Haematol 2005; 27: 307-11. Disponible en www.pubmed.com (consultado el 8 de noviembre de 2012).

22. Hernández JM, Martín G, Gutiérrez NC, Cervera J, Ferro MT, Calasanz MJ, et al. PETHEMA Cooperative Group, Spain Additional cytogenetic changes do not influence the outcome of patients with newly diagnosed acute promyelocytic leukemia treated with an ATRA plus anthracyclin based protocol. A report of the Spanish group PETHEMA. Haematologica 2001 aug; 86 (8): 807-13. Disponible en www.pubmed.com (consultado el 8 de noviembre de 2012).

23. Jácomo RH, Melo RA, Souto FR, de Mattos ER, de Oliveira CT, Fagundes EM, et al. Clinical features and outcomes of 134 Brazilians with acute promyelocytic leukemia who received ATRA and anthracyclines. Haematologica 2007 oct; 92 (10): 1431-2. Disponible en www.pubmed.com (consultado el 8 de noviembre de 2012).

24. Nucifora E, Fanal D, Goldstein S, Kusminsky G. Acute promyelocytic leukemia: experience with trans-retinoic acid in Argentina. Medicina 1996; 56: 333-8. Disponible en www.pubmed.com (consultado el 8 de noviembre de 2012).

25. Ruiz-Arguelles GL, Morales-Toquero A, Gómez-Rancel JD, López- Martínez B. Ruiz-Delgado GJ, Reyes-Núñez V. Treatment of acute promyelocytic leukemia: a single institution experience. Rev Invest Clin 2005; 57: 415-9. Disponible en www.pubmed.com (consultado el 8 de noviembre de 2012).

26. Avvisati G, Petti MC, Lo-Coco F, Vegna ML, Amadori S, Baccarani $\mathrm{M}$, et al. Induction therapy with idarrubicin alone significantly influences event-free survival duration in patients with newly diagnosed hypergranular acute promyelocytic leucemia: final results of the GIMEMA randomized study LAP 0389 with 7 years of minimal follow-up. Blood 2002; 100: 3141-6. Disponible en www.pubmed.com (consultado el 8 de noviembre de 2012).

27. Ades L, Sanz MA, Chevret S, Montesinos P, Chevallier $\mathrm{P}$, Raffoux E, et al. Treatment of newly diagnosed acute promyelocytic leukemia (APL): a comparison of FrenchBelgian-Swiss and PETHEMA results. Blood $2008 \mathrm{feb}$ 1; 111: 1078-84. Epub 2007 nov 1. Disponible en www. pubmed.com (consultado el 8 de noviembre de 2012).

28. Sanz MA, Lo Coco F, Martín G, Avvisati G, Rayón C, Barbui T, et al. Definition of relapse risk and role of nonanthracycline drugs for consolidation in patients with acute promyelocytic leukemia: a joint study of the PETHEMA and GIMEMA cooperative groups. Blood 2000 aug 15; 96 (4): 1247-53. Disponible en www.pubmed.com (consultado el 8 de noviembre de 2012).

29. Sanz M, Tallman M, Lo Coco F. Tricks of the trade for 
Leucemia promielocítica aguda. Resultados del PANDA - M. S. Undurraga et al

the appropriate management of newly diagnosed acute promyelocytic leukemia. Blood 2005; 105: 3019-25. Disponible en www.pubmed.com (consultado el 8 de noviembre de 2012).

30. Sanz MA. Recent advances in the treatment of APL. Clin Adv Hematol Oncol 2006; 4 (10): 727-9. Disponible en www.pubmed.com (consultado el 8 de noviembre de 2012).
31. Rego EM, Kim HT, Ruiz-Argüelles GJ, Uriarte Mdel $\mathrm{R}$, Jacomo RH, Gutiérrez-Aguirre H, et al. The impact of medical education and networking on the outcome of leukemia treatment in developing countries. The experience of International Consortium on Acute Promyelocytic Leukemia (IC-APL). Hematology. 2012 Apr; 17 Suppl 1: S36-8. Disponible en www.pubmed. com (consultado el 8 de noviembre de 2012). 